\title{
REPERCUSIONES DEL TEMOR AL CONTAGIO POR COVID-19 EN LA SALUD MENTAL DE MUJERES TRABAJADORAS EMBARAZADAS EN ECUADOR
}

\section{REPERCUSSIONS OF THE FEAR FROM CONTAGION BY COVID-19 IN THE MENTAL HEALTH OF PREGNANT WORKING WOMEN IN ECUADOR}

\author{
Sandra Barral Coral ${ }^{1}$ \\ Guido Albán Pérez²
}

Recibido: 2020-09- 25 / Revisado: 2020-10-09 / Aceptado: 2020-11-01 / Publicado: 2021-01-01

Forma sugerida de citar: Barral-Coral, S. y Albán-Pérez, G. (2021). Repersusiones del temor al contagio por COVID-19 en la salud mental de mujeres trabajadoras embarazadas en Ecuador. Retos de la Ciencia. 5(10), pp. 1-13. https://doi.org/10.53877/rc.5.10.20210101.01

\section{RESUMEN}

El temor al contagio por COVID-19 en mujeres embarazadas y sus posibles consecuencias en las emociones, conductas y temores propios del embarazo ha sido un tema poco abordado desde la perspectiva laboral. La presente investigación tuvo como finalidad describir la relación y establecer la inferencia que ha tenido el temor al contagio sobre la salud mental de las mujeres trabajadoras embarazadas en Ecuador. El estudio fue transversal con alcance correlacional. Se desarrolló un cuestionario de 25 preguntas que fue aplicado durante los controles prenatales a 48 mujeres. Los resultados muestran que existe relación entre el temor al contagio y la salud mental, la cual fue medida a través de la presencia de desórdenes emocionales, de la cantidad de sueño, en la percepción de mayor seguridad del parto en casa, su preferencia como lugar de parto y en el incremento de los temores propios del embarazo, especialmente aquellos relacionados con enfermedades o malformaciones en los neonatos y a no poder alimentarlos. Se ha confirmado lo encontrado en otros países como consecuencias del temor al contagio en las mujeres gestantes. Los resultados sugieren la necesidad de incluir a psicólogos en los servicios médicos ocupacionales que aborden estas manifestaciones con un enfoque salutogénico y preventivo.

Palabras clave: COVID-19, embarazo, temor, trabajo.

\section{ABSTRACT}

The fear of contagion by COVID-19 in pregnant women and its possible consequences on the emotions, behaviors and fears of pregnancy has been a subject little

\footnotetext{
${ }^{1}$ Magíster en Seguridad y Prevención de Riesgos del Trabajo. Profesora de la Universidad Central del Ecuador. Ecuador. Email: barralcoral@hotmail.com / ORCID: https://orcid.org/0000-0002-4675-9691

2 Ph.D. en Psicología. Profesor de la Universidad Central del Ecuador. Ecuador. Email: guidoalban62@hotmail.com / ORCID: https://orcid.org/0000-0003-4893-0023
} 
approached from the labor perspective. The purpose of this research was to describe the relationship and establish the inference that fear of contagion has had on the mental health of pregnant working women in Ecuador. The study was cross-sectional with correlational scope. A 25-question questionnaire was developed that was applied during prenatal check-ups to 48 women. The results show that there is a relationship between fear of contagion and mental health, which was measured through the presence of emotional disorders, of the amount of sleep, in the perception of greater safety of home birth, its preference as a place of childbirt and in the increase of fears of pregnancy, especially those related to diseases or malformations in neonate and not being able to feed them. What has been found in other countries has been confirmed as consequences of the fear of contagion in pregnant women. The results suggest the need to include psychologists in occupational medical services that treat these manifestations with a salutogenic and preventive approach.

Keywords: COVID-19, fear, job, pregnancy.

\section{INTRODUCCIÓN}

La COVID-19 ha visibilizado que ningún sistema de salud está preparado para enfrentar una enfermedad de esa naturaleza y magnitud. Al ser una enfermedad nueva, también se encuentran en desarrollo los estudios que muestran alternativas de prevención, tratamiento y la información disponible que permita conocer todas las consecuencias que se derivan de esta enfermedad, no solamente en la salud biológica sino además en la salud mental de toda la población y particularmente de mujeres gestantes que conservaron su trabajo durante la pandemia.

La Comisión Interamericana de Mujeres, (CIM) considera que el reto que tienen los gobiernos y organismos internacionales para encaminar políticas que busquen la igualdad en la mitigación y recuperación de esta crisis, podrá abrir una oportunidad en la medida en que respondan a las necesidades diferenciadas de la población, enfocándose en las necesidades de las mujeres. La CIM prioriza estudios sobre la salud de las mujeres, con especial énfasis en la salud sexual y reproductiva, por lo que presenta argumentos que avalan la incorporación de las necesidades de las mujeres en las decisiones, como una prioridad en los tiempos excepcionales que estamos viviendo. (OEA, CIM, 2020)

Henrietta Fore, Directora Ejecutiva de UNICEF, menciona que alrededor de 116 millones de neonatos nacerán bajo la sombra de la pandemia de la COVID-19. Las nuevas madres deben prepararse para traer una vida a un mundo totalmente distinto, en el que temen ir a los centros de salud por miedo a infectarse y en el que no pueden recibir cuidados de emergencia debido a la sobrecarga de los servicios de la salud, los aislamientos y a una falta de parteras cualificadas, ya que los trabajadores sanitarios, incluidas las matronas, están siendo reubicados para tratar a los pacientes con COVID-19, finaliza diciendo que "es difícil hacerse una idea de hasta qué punto la pandemia del coronavirus ha transformado la maternidad" (UNICEF, 2020).

La salud ocupacional, reconoce la exposición laboral a factores de riesgo como causa de accidentes y enfermedades relacionadas con el trabajo. No es novedad la interacción de factores de riesgo laborales con la salud mental de los trabajadores, así se tiene la exposición a ruido continuo, de impacto, vibraciones, a temperaturas extremas, disolventes volátiles, posturas forzadas, iluminación insuficiente 0 excesiva, trabajos monótonos, con sobrecarga mental o presión de tiempo, y entre los riesgos de tipo biológico ahora se añade la COVID-19. 
Respecto del estrés, como lo señala Damasio (1994) y Goleman (1995), citados en la Enciclopedia de salud y seguridad en el trabajo (OIT,1998), "la respuesta emocional es básica, pues el problema inicial de estrés suele ser lo que lleva a estados emocionales desagradables, como la ansiedad, el miedo y la depresión [...] la emoción es un determinante subyacente de la fuerza de las pautas de comportamiento, de la memoria y la cognición". Niveles altos de estrés aceleran el proceso de desgaste del organismo y si se mantienen en intensidad o duración, puede volverse un problema crónico o ser precursor de una enfermedad.

En lo que corresponde a políticas y normativas del Ecuador, los Art. 35 y 43 de la Constitución de la República del Ecuador (CRE), priorizan la protección, atención y cuidado de la salud integral de mujeres embarazadas, impulsando campañas para que acudan a controles médicos periódicos. Los Art. 32, 33 y 326 señalan al derecho a la salud, a un trabajo saludable y en un ambiente que garantice su salud, integridad y seguridad, mientras que el Art. 363 numeral 6, determina que el Estado será responsable de asegurar acciones y servicios de salud para garantizar la vida y salud de las mujeres embarazadas (Asamblea Nacional Constituyente, 2008).

La Organización de las Naciones Unidas (ONU), en su Objetivo de Desarrollo Sostenible № 3 sobre la salud y bienestar, indica que la COVID-19 está propagando el sufrimiento humano y destaca la necesidad de invertir en servicios públicos vitales. Su Objetivo del Milenio № 5 -relacionado a la salud materna- y cuya finalidad es garantizar la protección de los derechos humanos en el parto, incluye en ellos a las necesidades emocionales de la mujer gestante, ya que estas influirán en el futuro del neonato en lo psicológico y social (Fondo de Población de las Naciones Unidas, s.f.).

El artículo 2 del Instrumento Andino de Seguridad y Salud en el Trabajo, menciona que las normas relativas a esta temática deben regular las acciones en los lugares de trabajo para disminuir o eliminar los daños a la salud del trabajador; y el artículo 4, dictamina prevenir daños en la integridad física y mental de los trabajadores que sean consecuencia, guarden relación o sobrevengan durante el trabajo. (Comunidad Andina, 2004).

La Ley Orgánica de la Salud en su artículo 6, muestra que es responsabilidad del Ministerio de Salud Pública (MSP) -como rector en salud- el regular y vigilar las condiciones ambientales en las que desarrollan sus actividades los trabajadores; el artículo 118 ordena a los empleadores a proteger la salud de sus trabajadores, prevenir, disminuir o eliminar riesgos, accidentes y la aparición de enfermedades ocupacionales (Congreso Nacional, 2006).

Por su parte, la Ley de Seguridad Social establece la creación del Seguro General de Riesgos del Trabajo, el cual protege al afiliado y al empleador con programas de prevención de riesgos laborales y reparación de daños derivados de accidentes de trabajo y enfermedades profesionales, lo que incluye rehabilitación física, mental y reinserción laboral (Congreso Nacional, 2001).

El Reglamento de seguridad y salud de los trabajadores y mejoramiento del medio ambiente de trabajo, Decreto Ejecutivo 2393, en su artículo 66 de los riesgos biológicos, establece que los trabajadores expuestos a virus nocivos para la salud entre otros- deberá ser protegido en la forma indicada por la ciencia médica y la técnica en general (Presidencia de la República del Ecuador, 1986).

La Resolución C.D. 513 del Consejo Directivo del Instituto Ecuatoriano de Seguridad Social (IESS) contiene un listado de enfermedades profesionales clasificadas en: aquellas causadas por la exposición a agentes de las actividades 
laborales, las causadas por agentes químicos, físicos o biológicos y enfermedades infecciosas o parasitarias (IESS, 2016).

EI MSP, cuenta con la Política Nacional de Salud en el Trabajo 2019-2025, la cual define a las enfermedades en el trabajo como patologías ocasionadas por las condiciones de trabajo, las cuales pueden ser "específicas" si se relacionan directamente con el trabajo, e "inespecíficas" si no son determinadas por el trabajo, pero sí agravadas por este. Así también, señala que la exposición a factores de riesgo biológico facilita la probabilidad de causar un accidente de trabajo con material biológico infectado y que la salpicadura de fluidos es uno de los mecanismos de trasmisión (MSP, 2019).

La Organización Panamericana de la Salud (OPS) en su Plan de Acción sobre la Salud de los Trabajadores 2015-2025, establece objetivos que promueven el bienestar y salud de la población trabajadora, con énfasis en aquellos que están expuestos a condiciones de trabajo peligrosas. Todo lo cual establece un amplio marco regulador para la seguridad y la salud ocupacional en nuestro país.

Las consecuencias de la COVID-19 en el embarazo han sido descritas por la OPS y la Organización Mundial de la Salud (OMS) (2020), entre ellas se cita una menor asistencia a controles prenatales, posible detección tardía de problemas en el embarazo 0 en el feto que pueden poner en riesgo la vida de ambos; realidad compartida por países como Bolivia (Vargas 2020), España (Espartero, 2020) y Argentina (Romero, 2020). UNFPA (2020), menciona que mujeres embarazadas de Panamá, Egipto y Madagascar comparten los mismos temores de contagio y ansiedad respecto de la COVID-19.

Si bien es cierto que la COVID-19 puede provocar manifestaciones neurológicas, apoplejías que aumentan el riesgo de hospitalización, pérdida del olfato y/o el gusto, no es menos importante que el temor al contagio en el núcleo familiar, el aislamiento con la consecuente pérdida del contacto social presencial, familiar ampliado y laboral, así como la violencia intrafamiliar, afecten a la salud mental y al desarrollo del embarazo debido a la escasa actividad física al aire libre, estimulación intelectual, entre otros, que producen estrés y angustia en las mujeres embarazadas.

Respecto de las emociones en el embarazo, Cotarelo, Reynoso, Solano, Hernández y Ruvalcaba, en su estudio realizado en 85 mujeres embarazadas del Estado de Hidalgo de México, concluyen que "el miedo es la emoción más impactante ante la crisis por COVID-19, las gestantes afirman que la pandemia las ha despojado de cualquier emoción positiva, conduciéndolas así al miedo de contagiarse, la incertidumbre y la pena" (2020, p. 898).

Igartua indica, "si una embarazada está muy asustada y con miedo, su cerebro segregará cortisol, hormona del estrés, y ese cortisol también se eleva en el bebé. Para el bebé será mucho más complejo bajar esos niveles de cortisol si no los regula mamá [...] muchos niños que tienen rasgos ansiosos e inquietos fueron expuestos durante el embarazo a un alto nivel de cortisol", el transmitir al neonato ese temor, puede afectar su salud hasta después del nacimiento (2020, párrs. 1 y 4).

Los hallazgos determinados en una investigación realizada en mujeres embarazadas de China muestran que el miedo y la depresión fueron los eventos más encontrados, esto fue confirmado por Xiu-Min Jiang, enfermera del Hospital de Maternidad y Salud Infantil de Fujian, quien señaló "Si a una mujer embarazada se le diagnostica o se sospecha que tiene una infección por COVID-19, puede inducir diferentes grados de estrés psicológico, como miedo y ansiedad, que no favorecerían la salud de la madre o el niño" (Qing - Xiang Zheng RN. et al. 2020). 
A esto se suma otro temor más preocupante para la mujer gestante: la sensación de inseguridad al acudir a un centro médico para sus chequeos prenatales y al momento del parto en una maternidad $u$ hospital, esto ha ocasionado que algunas de ellas prefieran alumbrar en su propia casa tanto en España (Franco, 2020), New York, Estados Unidos (Kimiko, 2020), Colombia (Quintero, 2020) y México (NotiMex, 2020).

Un informe del Ministerio de Salud Pública (MSP) sobre la cobertura de control prenatal, mencionó que en comparación con el período enero-septiembre 2019, durante el mismo periodo de referencia en 2020 , se redujo la cobertura en el $22,21 \%$, la cual se atribuye a la emergencia sanitaria. Sobre los partos atendidos, hubo una reducción del 11,73\% en comparación con el 2019 y señala que las causas están en relación directa con la emergencia sanitaria y con una disminución en la proyección de gestantes para el presente año (MSP, 2020), lo cual coincide con lo que están viviendo en otros países como Bolivia (Vargas 2020), España (Espartero, 2020) y Argentina (Romero, 2020).

Al ser la gestación un período de singulares características y riesgos, se planteó investigar las repercusiones que podrían existir en la salud mental ante la presencia de temor al contagio por COVID-19, tanto sobre las emociones, comportamientos, percepciones, así como sobre los temores propios del embarazo en mujeres trabajadoras del Ecuador, para esto y en base a la revisión bibliográfica citada, se desarrolló y aplicó un cuestionario a las mujeres que acudieron a controles prenatales en tres centros médicos públicos del país.

Los datos confirman lo hallado por organismos internacionales en otros países sobre el temor de las mujeres embarazadas, sus efectos en la salud mental y la percepción de inseguridad en los hospitales públicos, lo cual ha incidido en que algunas de las mujeres prefieran tener su parto en casa, esto hace necesario que los servicios médicos ocupacionales de las empresas que están obligadas a tenerlos, incluyan en su cartera de servicios la atención psicológica tanto en promoción, prevención, así como la vigilancia de estas problemáticas, a fin de garantizar la protección a la salud mental de todos los trabajadores y en particular de las mujeres gestantes.

\section{MÉTODOS Y MATERIALES}

Se determinó para esta investigación un diseño no experimental de tipo transversal y un alcance correlacional. La población meta fueron mujeres embarazadas de las diferentes regiones del país que estuvieran trabajando durante la pandemia. Se seleccionó un servicio de salud público de primer nivel por región, a fin de encuestar a las mujeres gestantes durante sus controles prenatales en el mes de noviembre de 2020 , con lo cual, se consiguió una muestra de $n=48$.

Los criterios que se utilizaron para esta investigación fueron:

Criterios de inclusión

- Mujeres gestantes que conservaron su trabajo durante la pandemia

- Asistencia a controles prenatales en un centro público de atención ambulatoria

- Posesión de celular y acceso a internet que permita el despliegue de la encuesta Criterios de exclusión

- Encuestas llenadas fuera de la fecha establecida como límite de aplicación.

- Mujeres embarazadas que no desearon participar en la investigación

- Mujeres gestantes sin trabajo al momento del control prenatal

Criterios de exclusión. 
- Encuestas que no hayan sido llenadas correctamente

- Encuestas incompletas realizadas por las mujeres participantes en la investigación

- Mujeres embarazadas que no hayan firmado el consentimiento informado

Se realizó una revisión bibliográfica que fundamentó el diseño de un cuestionario de medición, para lo cual se definió como variable independiente el temor al contagio por COVID-19 y como variable dependiente la inferencia de este temor en la salud mental de las mujeres gestantes trabajadoras. El cuestionario contó con 25 preguntas de opción múltiple, las cuales se clasificaron en 3 categorías:

- Sociodemográfica: se preguntó por la edad, nivel de estudios completado, tiempo de embarazo y modalidades de trabajo.

- Temor al contagio por COVID-19: se indagó el temor personal al contagio en el centro de trabajo, durante el chequeo prenatal y el temor familiar al contagio de la mujer embarazada en los lugares citados.

- Repercusiones del temor: se buscaron alteraciones emocionales, de estado de ánimo, cogniciones erróneas, somatizaciones, incremento en los temores propios del embarazo, cambio en conductas como disminución de controles prenatales y preferencia del lugar para el parto.

El cuestionario elaborado fue revisado por tres expertos y posteriormente piloteado en mujeres embarazadas que no fueron parte del estudio. La versión final obtuvo un Alpha de Cronbach de 0,88 y fue aplicada online a través de un link enviado a los celulares de las mujeres gestantes durante la consulta prenatal. Para el análisis de los datos se estableció como significativo $p=0,05$, se utilizó el programa estadístico SPSS versión 22, y se aplicaron las pruebas Anova, Correlación de Spearman y Odds Ratio.

\section{RESULTADOS}

Dentro de la categoría sociodemográfica se obtuvieron los siguientes datos sobre los grupos de edad a los que pertenecieron las mujeres sujetas de este estudio:

\section{Gráfico 1:}

Edad de las mujeres embarazadas trabajadoras encuestadas

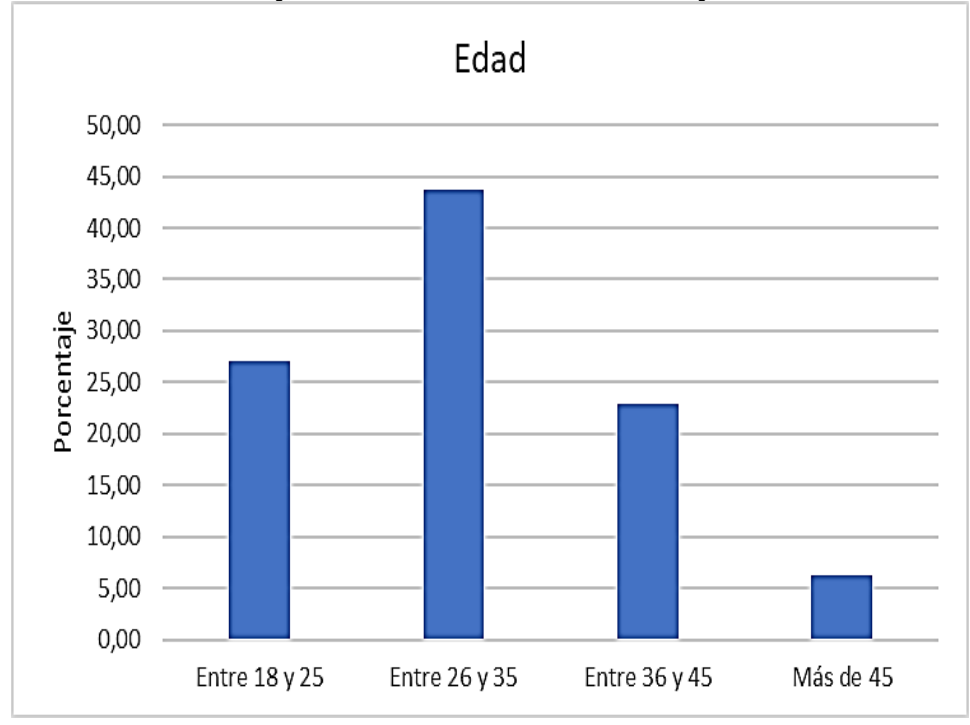

Fuente: Estudio de las repercusiones del temor al contagio por COVID-19 en la salud mental de mujeres trabajadoras embarazadas del Ecuador.

Elaborado por: elaboración propia 
La distribución de la muestra, en función de la ubicación de las mujeres encuestadas mostró lo siguiente:

\section{Gráfico 2:}

Región en la que viven las mujeres embarazadas trabajadoras encuestadas

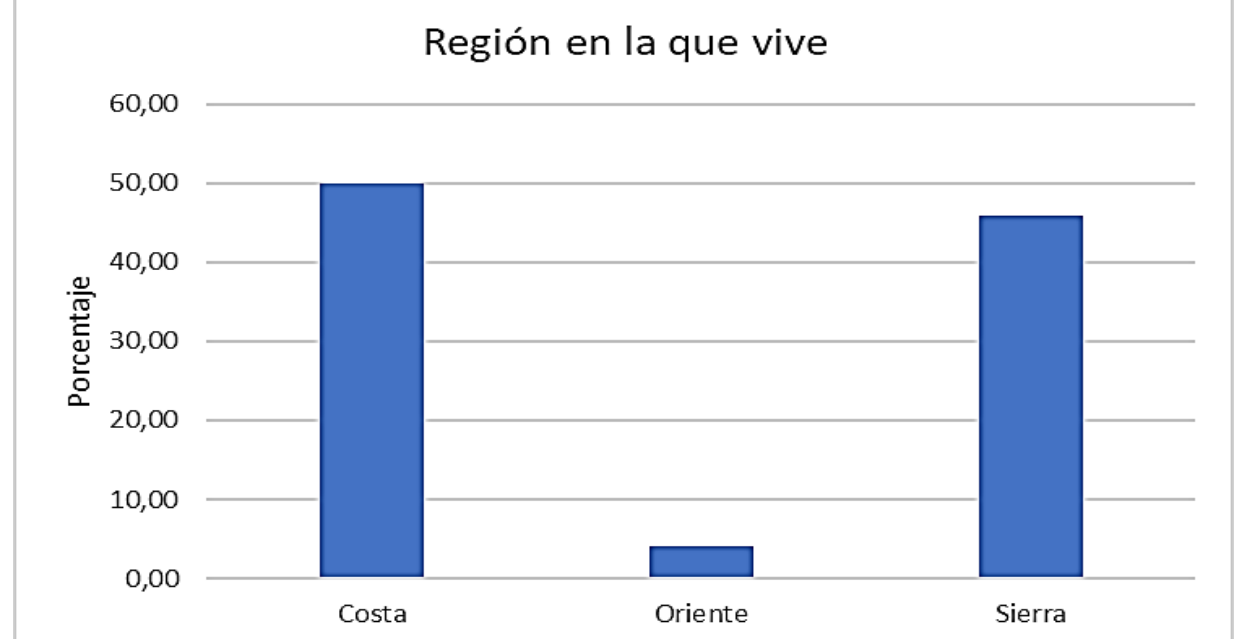

Fuente: Estudio de las repercusiones del temor al contagio por COVID19 en la salud mental de mujeres trabajadoras embarazadas del Ecuador Elaborado por: elaboración propia

Respecto de los niveles de estudios completados por las mujeres encuestadas se encontró:

Gráfico 3:

Nivel de estudio completado por las mujeres encuestadas

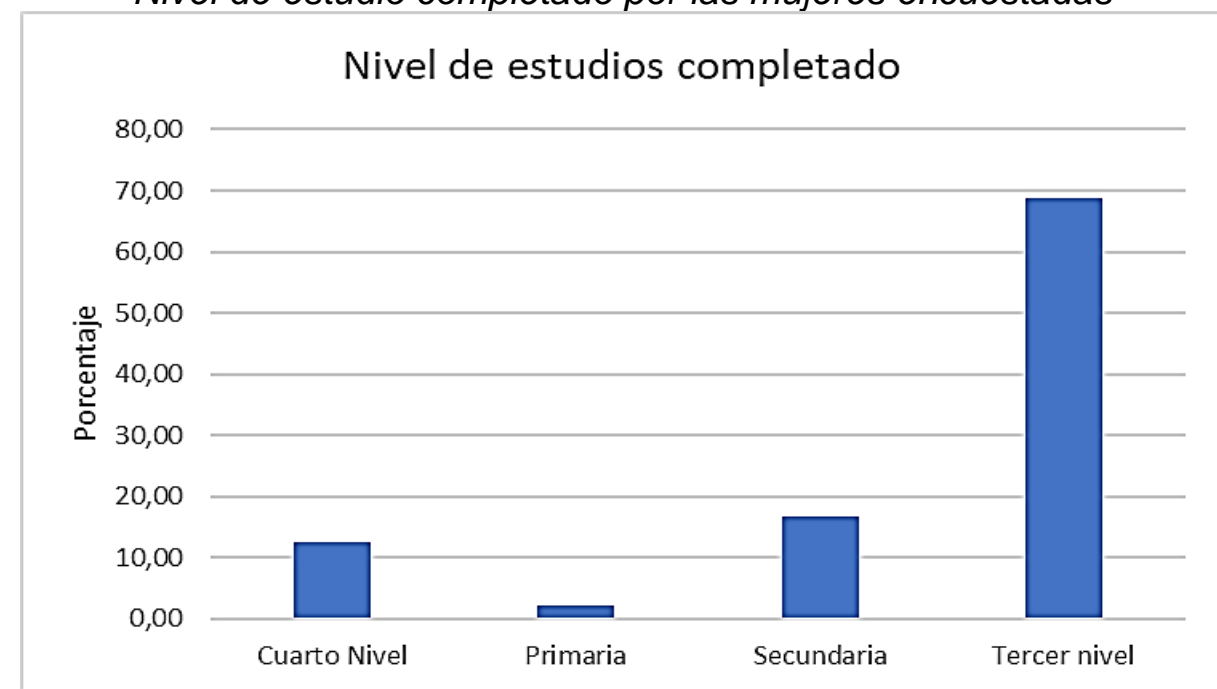

Fuente: Estudio de las repercusiones del temor al contagio por COVID-

19 en la salud mental de mujeres trabajadoras embarazadas del

Ecuador

Elaborado por: elaboración propia

Los tiempos de embarazo, de las mujeres encuestadas se distribuyeron de la siguiente manera: 
Gráfico 4:

Tiempo de embarazo de las mujeres encuestadas

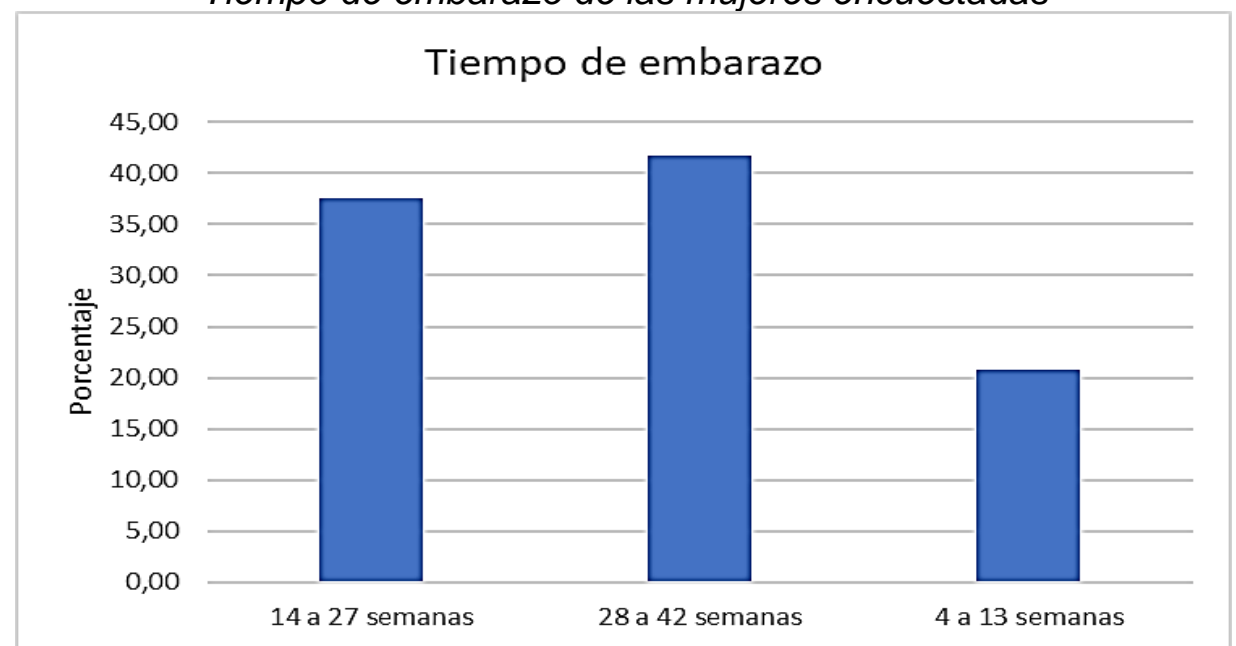

Fuente: Estudio de las repercusiones del temor al contagio por COVID19 en la salud mental de mujeres trabajadoras embarazadas del Ecuador Elaborado por: elaboración propia

Las modalidades de trabajo que realizaron estas mujeres fueron:

Gráfico 5:

Modalidad de trabajo de las mujeres encuestadas de acuerdo con la región en la que viven

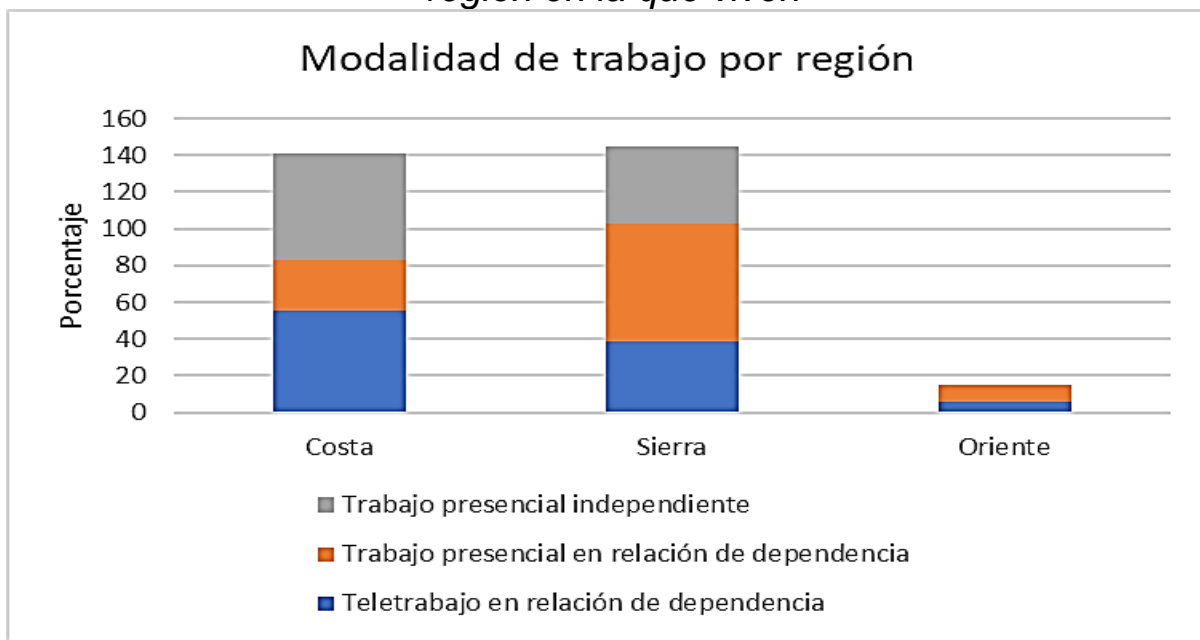

Fuente: Estudio de las repercusiones del temor al contagio por COVID19 en la salud mental de mujeres trabajadoras embarazadas del Ecuador Elaborado por: elaboración propia

En la aplicación de la prueba Anova se detectaron diferencias estadísticamente significativas entre las medias de 13 de los 20 indicadores de las variables medidas, siendo más llamativas: el aumento de temores propios del embarazo por temor a la COVID-19 F $(5,42)=11,718, p=, 000 ; y$ de estos, el temor a enfermedad 0 malformación en el/la bebé $\mathrm{F}(5,42)=18,046, p=, 000$; temor a no poder alimentar al/a la bebé $F(5,42)=7,759, p=, 000$ y temor a muerte del/la bebé $F(5,42)=7,057$, $p=, 000$. De los indicadores de somatización, el único con significancia estadística fue: desórdenes en la cantidad de sueño desde la pandemia $F(5,42)=2,660 p=, 035$. 
A fin de establecer la relación entre las variables estudiadas se aplicó la prueba Correlación de Spearman, la cual mostró que esta existe con 15 de los 17 indicadores de la variable dependiente, siendo las más fuertes con el temor a enfermedad o malformación en el/la bebé $\rho=, 775$, temor a no poder alimentar al/a la bebé $\rho=, 686$ y temor a muerte del/la bebé $\rho=, 630$, todas con significancia bilateral de, 000 . Los dos únicos indicadores con los que no se encontró relación significativa fueron: percepción falsa de síntomas de COVID-19 y disminución de controles prenatales por pandemia.

La fuerza de asociación fue medida mediante la aplicación de la prueba Odds Ratio $(\mathrm{OR})$, la cual arrojó los siguientes resultados:

Tabla 1:

OR del temor al contagio por COVID-19 en la salud mental de mujeres trabajadoras embarazadas ODDS RATIO

\begin{tabular}{lc}
\hline \multicolumn{1}{c}{ Repercusiones del temor } & TOTAL Temor por COVID-19 \\
\hline Percepción falsa de síntomas de COVID-19 & 0,880 \\
Desórdenes emocionales/estado de ánimo por temor a contagio de COVID-19 & 3,068 \\
Desórdenes de cantidad de sueño desde la pandemia & 5,556 \\
Desórdenes de contenido de sueño desde la pandemia & 0,882 \\
Disminución de controles prenatales por pandemia & 1,760 \\
Falso trabajo de parto por temor a COVID-19 & 0,000 \\
Preferencia de parto en casa por temor a COVID-19 & 2,438 \\
Percepción de seguridad del parto en casa durante la pandemia & 2,333 \\
Temor al contagio de COVID-19 aumenta temores del embarazo & 63,000 \\
Temor a complicaciones durante el embarazo o parto & 6,344 \\
Temor a tener un parto prematuro & 1,971 \\
Temor a sentir dolor en el parto & 2,875 \\
Temor a no poder dar a luz & 2,297 \\
Temor a la cesárea & 3,500 \\
Temor a enfermedad o malformación en el/la bebé & 10,636 \\
Temor a muerte del/la bebé & 3,733 \\
Temor a no poder alimentar al/a la bebé & 14,667 \\
\hline Fuente: Estudio de las repercusiOnes del temor al contagiO pOr COVIDD-19 en
\end{tabular}

Fuente: Estudio de las repercusiones del temor al contagio por COVID-19 en la salud mental de mujeres trabajadoras embarazadas del Ecuador

Elaborado por: elaboración propia

Lo observado indica que existe entre 2 y 63 probabilidades más de que se presenten desórdenes emocionales y de estado de ánimo por temor a sufrir contagio de COVID19, mientras que la cantidad de sueño, el cambio en la preferencia de lugar de parto, la percepción de seguridad sobre el mismo y el aumento de temores propios del embarazo ante el temor al contagio por COVID-19, son cada vez más frecuentes en las mujeres embarazadas, lo que hace que esta etapa de gestación esté llena de temores y complicaciones psicológicas.

\section{DISCUSIÓN}

El embarazo como etapa de transformaciones tanto físicas como emocionales para las mujeres, requiere de adaptaciones rápidas a los cambios que experimentan y a las progresivas necesidades, la presencia de la COVID-19 en el país y el temor a su contagio, ocasionó en las mujeres trabajadoras encuestadas algunos desórdenes en 
las emociones, en la cantidad de sueño, en que consideren más seguro el parto en casa e incrementó temores propios del embarazo, específicamente a enfermedades o malformaciones en los neonatos, a no poder alimentarlos y a complicaciones durante el embarazo o parto.

Las manifestaciones emocionales más comunes halladas en este estudio fueron: angustia, irritabilidad, llanto fácil, tristeza periódica, tensión emocional y muscular, lo cual confirma la afectación emocional encontrada por Cotarelo, Reynoso, Solano, Hernández y Ruvalcaba en su estudio realizado en México (2020, p. 898) y por el estudio realizado en China (Qing - Xiang Zheng RN. y otros, 2020), esto puede entenderse también debido a las reacciones químicas cerebrales desencadenadas por el temor, las cuales exacerban la producción de vasopresina por sobre la oxitocina (ambas producidas por la amígdala cerebral), así como adrenalina y cortisol, debido a la presencia de estrés.

La mayoría de las mujeres encuestadas manifestaron sentir temor a contagiarse durante el chequeo prenatal y más de la mitad de sus familias comparten ese temor, dato que se relaciona con lo señalado por Franco (2020), esto ha incidido en la percepción de inseguridad al momento del parto, lo cual confirma lo encontrado por el mismo autor, Kimiko, Quintero, NotiMex, así como por la UNICEF (2020). Este temor puede verse influido e incrementado, además por la cantidad de información errada, sin verificación ni credibilidad, que de forma irresponsable difunden ciertos medios de comunicación, especialmente los que no están sujetos a las regulaciones nacionales.

No se ha podido visibilizar una relación estadísticamente significativa entre el temor al contagio por COVID-19 y la percepción errónea de síntomas que fueran confundidos con la enfermedad citada, falso trabajo de parto y desórdenes en el contenido del sueño; mientras que la disminución en los controles prenatales señalados por el Ministerio de Salud Pública (2020)- no obedecen únicamente al temor al contagio como tal, se han indicado como causas adicionales la enfermedad del profesional de salud, cierre de la unidad médica, dificultades económicas, temor al contagio en el medio de transporte o falta de este último, dato que también fue anotado por UNFPA (2020).

No todas las mujeres del estudio estuvieron en teletrabajo, la mayoría de mujeres embarazadas en modalidad presencial durante la pandemia fueron trabajadoras independientes, se deduce que probablemente no tuvieron otra opción; sin embargo, de las trabajadoras en relación de dependencia con trabajo presencial, el 45,45\% estaba en el primer trimestre gestacional, por lo cual quizás el embarazo aún no había sido detectado o informado al centro laboral para evitar posibles despidos, pero el $54,55 \%$ estuvo en el segundo trimestre gestacional, esto hace necesario investigar las razones por las que les negó su derecho constitucional a protección prioritaria.

El escenario mundial y el marco conceptual y normativo nacional respecto de la seguridad y salud laboral, ha mostrado la necesidad de incluir a la COVID-19 como accidente de trabajo y/o enfermedad ocupacional toda vez que la fuente de contagio puede estar en los centros hospitalarios donde labora el personal de salud, siendo así una patología laboral específica, o inespecífica si el contagio se ha producido por contacto con fluidos de alguien enfermo no detectado oportunamente o asintomático que continuó laborando en cualquier tipo de centro de trabajo.

Así también es menester incorporar al temor al contagio por COVID-19 en el trabajo, como factor de riesgo psicosocial emergente de tipo individual, ya que puede modificar conductas que pongan en riesgo la salud de los trabajadores, más aún en 
el caso de mujeres embarazadas, en las cuales este temor funciona como precursor del incremento de los temores propios del embarazo y en el descenso de los controles prenatales en algunas de ellas.

La afectación en la salud mental de las mujeres gestantes estudiadas, tanto en las emociones, comportamientos y percepciones, muestra la necesidad de incluir en los servicios médicos ocupacionales a Psicólogos clínicos que trabajen con estrategias de promoción de la salud, prevención de estas problemáticas y tratamiento oportuno de las mismas, para lo cual se requiere que el rector del trabajo actualice la norma que lo contempla e incorpore el puesto de psicólogo ocupacional en los manuales de descripción, valoración y clasificación de puestos.

Finalmente, es necesaria la aplicación de la encuesta en una muestra más amplia de mujeres trabajadoras, en servicios de salud de primer y segundo nivel tanto públicos como privados e incluir las funciones y puestos de trabajo que ocupan las mujeres sujetas de estudio, a fin de conocer el riesgo adicional durante el embarazo en estas circunstancias, fundamentar su inclusión en las normativas de seguridad y salud en el trabajo y poder tener una visión más cercana de lo que sucede en todo el país.

\section{REFERENCIAS}

Asamblea Nacional Constituyente. (2008). Constitución de la República del Ecuador. Quito, Ecuador: Ediciones Lexis.

Comunidad Andina. (2004). DECISIÓN 584: Instrumento Andino de Seguridad y Salud en el Trabajo.

Congreso Nacional del Ecuador. (2006). Ley Orgánica de Salud. Quito, Ecuador: Ediciones Lexis.

Congreso Nacional del Ecuador. (2001). Ley de Seguridad Social. Quito, Ecuador: Ediciones Lexis.

Cortés J. (abril, 2020). Por miedo al Covid-19, indígenas embarazadas buscan parteras. Diario El Heraldo de Juárez, México. Recuperado el 21 de octubre del 2020, de: https://www.elheraldodejuarez.com.mx/mexico/sociedad/por-miedo-al-covid-19indigenas-embarazadas-buscan-parteras-contagios-coronavirus-nacimientos5155233.html

Cotarelo, A., Reynoso, J., Solano, C., Hernández, M., y Ruvalcaba, J. (agosto, 2020). Impacto del COVID-19 en las emociones de mujeres embarazadas con diabetes gestacional. Journal of negative \& no positive results, 5(8), 766-913.

Diario El Comercio. (abril, 2020). El miedo a dar a luz en Guayaquil, una ciudad diezmada por la pandemia. El Comercio. Recuperado el 19 de octubre de 2020, de: https://www.elcomercio.com/actualidad/embarazo-mujeres-guayaquil-pandemiacoronavirus.html

Diario El Comercio. (mayo, 2020). La maternidad en medio del covid-19, se vive con sobresaltos. El Comercio. Recuperado el 19 de octubre de 2020, de: https://www.elcomercio.com/actualidad/maternidad-coronavirus-sobresaltos-partosmadres.html

Espartero, M. (marzo, 2020). Cortés J. (abril, 2020). Por miedo al Covid-19, indígenas embarazadas buscan parteras. Diario El Español, España. Recuperado el 13 de noviembre del 2020, de: https://www.elespanol.com/sociedad/20200325/panicohospital-covid-19-viven-embarazadas-salen-cuentas/477203954_0.html

Fondo de Población de las Naciones Unidas. (marzo de 2020). Comunicado del UNFPA sobre el nuevo coronavirus (COVID-19) y el embarazo. Fondo de Población de las Naciones Unidas. Recuperado el 20 de octubre de 2020, de: 
https://www.unfpa.org/es/press/comunicado-del-unfpa-sobre-el-nuevo-coronaviruscovid-19-y-el-embarazo

Fondo de Población de las Naciones Unidas. (s.f.). Quinto Objetivo de Desarrollo del Milenio: mejorar la salud materna. Fondo de Población de las Naciones Unidas. Recuperado el 20 de octubre de 2020, de: https://www.unfpa.org/es/quinto-objetivo-de-desarrollodel-milenio-mejorar-la-salud-materna

Franco, L. (mayo de 2020). El miedo al contagio por coronavirus aumenta el interés por los partos en casa. Diario El País. Recuperado el 19 de octubre de 2020, de: https://elpais.com/elpais/2020/05/21/mamas_papas/1590040313_226582.html

García, A. (agosto de 2020). Menos partos en el sistema público, asistencia municipal y áreas para embarazadas contagiadas durante la pandemia en Guayaquil. El Comercio. Recuperado el 19 de octubre de 2020, de: https://www.elcomercio.com/actualidad/partos-sistema-publico-municipioguayaquil.html

García, M. (agosto de 2020). "Sentía pánico todo el tiempo": El miedo de parir en una pandemia. El Confidencial. Recuperado el 15 de noviembre de 2020, de: https://confidencial.com.ni/sentia-panico-todo-el-tiempo-el-miedo-de-parir-en-unapandemia/

Igartua P. (junio, 2020). La experiencia del embarazo en el Covid-19. Diario EuropaSur. Recuperado el 19 de octubre del 2020, de: https://www.europasur.es/opinion/articulos/experiencia-embarazo-Covid19_0_1473452934.html

Instituto Ecuatoriano de Seguridad Social (2016). Resolución CD 513 Reglamento del Seguro General de Riesgos del Trabajo.

Kimiko. (junio, 2020). Coronavirus en NY: cada vez más mujeres prefieren parir en su casa. Diario Clarín. Recuperado el 14 de noviembre de 2020, de: https://www.clarin.com/new-york-times-international-weekly/coronavirus-ny-vezmujeres-prefieren-parir-casa_0_ZWIlv5uXv.html

Ministerio de Salud Pública. (2019). Política nacional de salud en el trabajo. Quito, Ecuador: Ministerio de Salud Pública.

Ministerio de Salud Pública. (2020). Respuesta ciudadana DNPNAS \#009. 26 de octubre de 2020.

NotiMex. (abril, 2020). Ante el temor de contraer Covid-19, embarazadas quieren partos en casa. Diario 24 horas. El diario sin límites. Recuperado el 13 de noviembre de 2020, de: $\quad$ https://www.24-horas.mx/2020/04/09/ante-el-temor-de-contraer-covid-19embarazadas-quieren-partos-en-casa/

OEA, CIM (2020). COVID-19 en la vida de las mujeres. Razones para reconocer los impactos diferenciados. pp.17-21.

Organización Internacional del Trabajo. (1998). Enciclopedia de Salud y Seguridad en el Trabajo. Capítulo 34, Factores Psicosociales y de Organización. pp. 2-62.

Organización de las Naciones Unidas. (s.f.). Objetivos de desarrollo sostenible. Objetivo 3: Garantizar una vida sana y promover el bienestar para todos en todas las edades.

Organización Panamericana de la Salud. (2015). Plan de Acción sobre la Salud de los Trabajadores 2015-2025.

Organización Panamericana de la Salud / Organización Mundial de la Salud. (agosto, 2020). Alerta Epidemiológica COVID-19 durante el embarazo-13 de agosto de 2020. Washington, D.C: Organización Panamericana de la Salud/Organización Mundial de la Salud.

Presidencia de la República del Ecuador. (1986). Decreto Ejecutivo 2393. Reglamento de seguridad y salud de los trabajadores y mejoramiento del medio ambiente de trabajo.

Ponce, T. (mayo, 2020). La difícil labor de parir en una pandemia. GK. Recuperado el 20 de octubre de 2020. 
Quintero, K. (junio, 2020). La partería en época de COVID-19. Diario El Espectador. Recuperado el 15 de noviembre de 2020, de: https://www.elespectador.com/noticias/nacional/parteria-durante-el-covid-19/

Qing - Xiang Zheng RN. et al., 2020). La influencia de la respuesta psicológica y el sentido de seguridad en el estrés del embarazo durante el brote de la enfermedad por coronavirus 2019: un modelo mediador. Journal of Clinical Noursing. Volumen 29. noviembre de 2020. Edición 21-22. pp. 4248 - 4257. Recuperado el 30 de noviembre de 2020 de: https://onlinelibrary.wiley.com/doi/10.1111/jocn. 15460

Romero, C. (mayo, 2020). Dudas, miedos y precauciones durante el embarazo en tiempos de Covid-19. Diario El milenio. Recuperado el 13 de noviembre de 2020, de: https://elmilenio.info/2020/05/14/dudas-miedos-y-precauciones-durante-el-embarazoen-tiempos-de-covid-19/

UNFPA (junio, 2020). Las indígenas embarazadas en Panamá enfrentan los temores de la COVID-19 y la falta de transporte. Recuperado el 26 de octubre de 2020, de: https://www.unfpa.org/es/news/las-ind\%C3\%ADgenas-embarazadas

UNICEF (mayo, 2020). Las mujeres embarazadas y los bebés nacidos durante la pandemia de la COVID-19 se enfrentarán a unos sistemas de salud sobrecargados y a interrupciones en los servicios. Recuperado de: https://www.unicef.org/es/comunicados-prensa/mujeres-embarazadas-y-bebesnacidos-durante-covid-19-enfrentaran-sistemas-salud-sobrecargados

Vargas, N. (julio, 2020). Embarazos en pandemia: controles en casa y prueba COVID-19 antes del parto. Diario Opinión. Recuperado el 13 de noviembre de 2020, de: https://www.opinion.com.bo/articulo/revista-asi/embarazos-pandemia-controles-casaprueba-covid-19-antes-parto/20200726015342779456.html 